\title{
Zur musikalischen Dramaturgie der Operetten bei Jacques Offenbach und Arthur Sullivan am Beispiel von Les Brigands und The Pirates of Penzance
}

\author{
von Ronny Porsch, Dreieich
}

Obwohl neuere Forschungsarbeiten andere Akzente gesetzt wissen wollen, ${ }^{1}$ herrscht innerhalb der Musikwissenschaft ein breiter Konsens darüber, dass am 5. Juli 1855 mit der Premiere der beiden Einakter La nuit blanche und Les deux aveugles von Jacques Offenbach im Théatre des Bouffes Parisiens zugleich die Geburtsstunde der Operette schlug. Weniger Klarheit herrscht hingegen über die Rezeption der Werke Offenbachs außerhalb Frankreichs und ihre prototypische Geltung für andere Komponisten des 'leichten Genres'. Der vorliegende Aufsatz sucht diese Lücke zu schließen, indem einem Werk Jacques Offenbachs eines von Arthur Sullivan gegenübergestellt wird, dem Hauptvertreter der englischen Spielart musikalischen Unterhaltungstheaters, welcher zusammen mit seinem Librettisten William S. Gilbert im letzten Drittel des 19. Jahrhunderts in London ähnliche Erfolge feiern konnte wie Offenbach in Paris. Als Anschauungsmaterial boten sich mit Les Brigands auf der einen und The Pirates of Penzance auf der anderen Seite zwei Stücke an, die beide im Räubermilieu angesiedelt sind und hinsichtlich ihrer Entstehung chronologisch nicht allzu weit auseinander liegen. Ausgehend von einleitenden Vorüberlegungen werden Libretto, Dramaturgie und Werkstruktur der jeweiligen Stücke einer vergleichenden Analyse unterzogen, um zu klären, ob und inwieweit eine gemeinsame Vorstellung über den Aufbau eines komischen Opernwerks vorlag und welches schließlich die Unterschiede im jeweiligen szenischen Verständnis sind.

So etwa Jacques Rouchouse: Hervé - le père de l'Opérette, Paris 2000. 


\section{Les Brigands}

\section{I.}

Irgendwo in Mantua

Um Falsacappa und seine Banditen steht es schlecht. Die Geschäfte laufen desaströs und die letzte Geisel, Fragoletto, ist nicht nur pleite, sondern darüber hinaus auch noch in des Banditenchefs Tochter Fiorella verliebt. Doch Fragoletto verspricht als tüchtiger Räuber die Banditen zu unterstützen, wenn man ihm die Hand Fiorellas gewährt. Gemeinsam macht man sich zu neuen Beutezügen auf. Fiorella bleibt im Lager zurück und begegnet plötzlich dem völlig konsternierten Herzog von Mantua, der sich verirrt hat. Sie schenkt ihm die Freiheit und weist ihm den richtigen Weg. Die fröhlich heimkehrenden Räuber haben einen Plan: Von einem gefangenen Kurier haben sie erfahren, dass der Herzog von Mantua eine hohe Belohnung an den Hof von Granada zahlen will, da er die dortige Prinzessin zu ehelichen gedenkt. Gemeinsam beschließen die Räuber, sich des Geldes zu bemächtigen, indem sie die Gesandtschaften beider Höfe überfallen und unter Vorspielung falscher Tatsachen als verkleidete Spanier beim Herzog von Mantua die Belohnung erschleichen.

Der Plan und die Aufnahme Fragolettos in den Kreis der Briganten wird anschließend gebührend gefeiert.

II.

Pipos Waldschänke

Die Räuber erscheinen am vereinbarten Treffpunkt beider Gesandtschaften und überfallen zunächst die Wirtsleute und danach die mantuanische Delegation in wechselnder Verkleidung. Auch die darauf erscheinende spanische Abordnung, angeführt von Gloria-Cassis, wird ohne größere Mühen überwältigt. Die Banditen bemächtigen sich deren Kleider und ziehen getarnt als 'falsche' Spanier an den Hof von Mantua.

\section{III.}

Hof von Mantua

In Erwartung seiner Vermählung feiert der junge Herzog von Mantua gemeinsam mit den Hofdamen seinen Junggesellenabschied. Schon nahen die verkleideten Banditen. Der Herzog vermeint zunächst Fiorella wiederzuerkennen, wird jedoch von der überraschenden Nachricht seines korrupten Finanzministers erschüttert, in der Staatskasse herrsche beklemmende Leere. Falsacappa ist außer sich, wähnt er als Meisterdieb sich doch inmitten einer infamen Intrige. Plötzlich erscheint die echte spanische Delegation, welche sich von ihren Fesseln im Wirtshaus befreien konnte und nun Falsacappas Mannen als Betrüger entlarvt. Doch der Herzog lässt unter dem Eindruck von Fiorellas Schönheit Gnade vor Recht ergehen, schenkt den Banditen die Freiheit und ernennt sie zu seinen Polizisten. 


\section{The Pirates of Penzance or the Slave of Duty}

\section{I.}

Die Küste vor Cornwall

Der 21jährige Vollwaise Frederic gibt seinen Ausstand bei den Piraten von Penzance. Die vom Branntwein berauschte Menge feiert das Ende seiner Piratenlehrzeit, die eigentlich ein Missverständnis war. Ruth, sein Kindermädchen, war instruiert, den Jungen als "pilote" ausbilden zu lassen, verstand jedoch aufgrund ihrer Schwerhörigkeit "pirate". Gleichwohl hegt die ergraute 'Frau für alle' die Hoffnung, Frederic würde ihr verzeihen und sie eines Tages ehelichen. Doch dieser lernt am ersten Tag in Freiheit gleich seine erste Liebe kennen. Es ist Mabel, Tochter aus gutem Hause und Schwester zahlreicher lokaler Schönheiten, die auf die übrigen Piraten einen enormen Eindruck machen. Da tritt Major-General Stanley auf den Plan, ein ausrangierter Diener Ihrer Majestät und zugleich Vater der hübschen Mädchen. Ihm gelingt es nicht nur mit Wortsalven die Piraten von seinen Fähigkeiten zu überzeugen, er rettet auch seine Töchter, indem er vorgibt, ein Waise zu sein, welchen die Korsaren niemals Gewalt antun.

\section{II.}

Eine verlassene Kapelle auf dem Land

Tiefe Gewissensbisse quälen den General ob seines unehrenhaften Verhaltens. Doch Frederic weiß schon einen Plan: Die Polizei Londons soll es richten und die Piraten verhaften. Freilich, der Plan schlägt fehl. Die gedemütigte Ruth hat den Piratenkönig darauf aufmerksam gemacht, dass Frederic aufgrund seines Geburtsdatums - 29. Februar eigentlich erst 5 1/4 Jahre alt sei, und folglich noch weitere Jahre seinen Piratendienst ableisten müsste. Der Piratenchef ist erstaunt. Frederic ist es auch, fügt sich aber als 'Sklave der Pflicht' willig in sein Schicksal. Mabel jedoch beschließt zu kämpfen. Auf ihr Geheiß erscheinen wirklich Londoner Bobbies, deren illustre Zahl freilich nicht darüber hinwegtäuschen kann, dass ihr Kopf leer und ihre Hosen voll sind. Die totale Niederlage gegen die wütenden Piraten kann gerade noch abgewendet werden, als der Sergeant auf eine List verfällt. Er beschwört feierlich den Namen Königin Viktorias, worauf sich die Piraten niederkniend ergeben. Sie alle sind nämlich Peers die vom rechten Weg abgekommen sind. Major-General Stanley obliegt es, Liebe vor Recht ergehen zu lassen - er gibt das Startsignal für eine finale Massenhochzeit. 


\section{I. $\quad$ Einleitung}

$-2-$

Die Frage, ob und inwieweit William S. Gilbert und Arthur Sullivan sich von Werken französischer Autoren inspirieren ließen, beantwortet ein Blick in die Werkverzeichnisse. So sind bei Gilbert drei der vier von ihm adaptierten Stücke als Werke Meilhacs und Halévys rubriziert. ${ }^{2}$ Auch Arthur Sullivans Werkkatalog weist in der Abteilung Bearbeitungen fremder Werke die Namen zahlreicher französischer Komponisten wie etwa DanielFrançois-Esprit Auber oder Charles Gounod auf. Offenbar schien die französische Oper, und hier insbesondere das Unterhaltungstheater, verkörpert in der Person Jacques Offenbachs, eine immense Attraktivität auszustrahlen, was nicht verwunderlich ist, wenn man den Erfolg seiner Werke auf den Bühnen Londons betrachtet. ${ }^{3}$ Dieser Erfolg schloss das hier behandelte Stïck mit ein. So erfuhr Les Brigands gleich zweimalig eine englische Bearbeitung. Einerseits durch Henry S. Leigh, dessen Falsacappa am 22. 4. $1871 \mathrm{im}$ St. James Theatre Premiere hatte, andererseits durch William S. Gilbert selbst, dessen eigene Version von Les Brigands aus dem Jahre 1871 mit Rücksicht auf die früher begonnene Übersetzungsarbeit Henry Leighs erst 1889 im Avenue Theatre zur Aufführung kam und einstweilen zur Sicherung der Urheberrechte nur pro forma veröffentlicht wurde. ${ }^{4}$

\section{$-3-$}

Mit Blick auf die Gattungsbezeichnungen der Stücke wird zugleich deutlich, dass die englischen Autoren nicht beabsichtigten Offenbachs dramatische Kunst lediglich affirmativ nachzuahmen, sondern vielmehr bestrebt waren einen eigenständigen Typus komischer Oper zu etablieren. Nicht zuletzt deshalb trägt ein Großteil ihrer Werke die Bezeichnung comic ope$r a{ }^{5}$ Damit ist zugleich eine Nähe zur großen Oper angesprochen, der sich

2 Außer Les Brigands waren dies The Realm of Joy (1873) nach Le roi candaule (1859) und The Bridge of Sighs (1872) nach Le Pont des soupirs (1861).

3 Vgl. Andrew Lamb: How Offenbach conquered London, in: Opera 20 (1969), S. 932-938.

4 Offenbachs Funktion als Wegbereiter für Gilbert und Sullivan in London war bedeutend. Sullivans erster Librettist, F.C. Burnand, hatte bereits Werke Offenbachs ins Englische übertragen. Zudem waren die ersten Gemeinschaftsproduktionen Gilberts und Sullivans oft in Ergänzung zu Stücken der Bouffes Parisiens gespielt worden. Vgl. dazu Andrew Lamb: Offenbach in London. Chronologie der Aufführungen seit 1857, in: Offenbach und die Schauplätze seines Musiktheaters, hrsg. von Rainer Franke (= Thurnauer Schriften zum Musiktheater 17), Laaber 1999, S. 200.

5 Die in einschlägigen Lexikaeinträgen (z.B. New Grove Dictionary of Music and Musicians) wiederkehrende Bezeichnung Operetta verwendeten sie dagegen nie. 
Sullivan als in Leipzig ausgebildeter Mendelssohn-Stipendiat eher verbunden fühlte als dem Boulevardtheater. Bei Offenbach hingegen erscheint die Etikettierung seiner Werke willkürlich. So finden sich neben bouffonerie, bouffonerie musicale, opérette, und opérette bouffe auch ausgefallenere Titel wie chinoiserie musicale und conversation alsacienne. Offenbar dienten die Bezeichnungen hauptsächlich dem Zweck, das humoristische Gepräge der Stücke anzudeuten und mit ihrer Originalität die Neugier der Zuschauer zu wecken. Les brigands firmiert dagegen unter der Bezeichnung Opéra bouffe.

Neben dem hieraus offensichtlichen Bezug zur italienischen Opera buffa war für Offenbach vor allem der Traditionszusammenhang mit der Opéra comique von Bedeutung, welcher er aufgrund vermehrten Einbezugs lyrischer und sentimentaler Elemente ein Vernachlässigen des Komödiantischen attestierte, und zu der er mit seinem von ihm geschaffenen Gattungsmodell des "genre primitif et vrai" Alternativen entwickeln wollte.

$-5-$

Trotz terminologischer Divergenzen verbindet die hier untersuchten Werke ihre satirische Grundhaltung, weshalb die Atmosphäre der Stücke insgesamt ironisch und gelöst ist. Satirisch behandelt wird vor allem die zeitgenössische Gesellschaft. So finden sich bei Offenbach Verweise auf das Paris seiner Zeit nicht nur in einzelnen Personen, wenn sich etwa die verstiegenen Hofschranzen Campotasso und Gloria-Cassis durch ihre Gebärden als getarnte Boulevardiers zu erkennen geben, sondern vor allem auch in der ausführlich dargestellten sexuellen Libertinage, als Zerrbild einer überliberalisierten Auffassung von Geschlechterbeziehungen innerhalb der großstädtischen Aristokratie und Bourgeoisie.

$-6-$

Auch Gilberts Libretto zeigt bestimmte Handlungskonstanten, die ihren Ursprung in der zeitgenössischen englischen Gesellschaft haben. Das sind neben den immer wiederkehrenden Bezügen zur Nautik und Monarchie vor allem typisch britische Themen wie moralische Integrität und Pflichtgefühl. Dabei binden sich die Protagonisten gewissenhaft an Vorgaben, Gesetze oder Versprechungen, seien sie auch noch so grotesk. So nimmt es Frederic in The Pirates of Penzance ebenso klaglos hin, seine Lehrzeit bei den Piraten aufgrund eines Versehens notfalls weitere 16 Jahre abzuleisten, wie die Piraten am Schluss schon bei der leisesten Andeutung des

6 Der oft zu lesende Ausdruck genre primitif et gai beruht vermutlich auf einem Lesefehler. Siehe das Original in: Jacques Offenbach. Lettres à Meilhac et Ludovic Halévy, hrsg. v. Philippe Goninet, Paris 1994, S. 275. 
Namens der Königin automatisch auf die Knie fallen. Für den Betrachter sind diese Bindungen deshalb amüsant, weil er deren irrationalen Charakter erkennt, während die handelnden Personen mechanisch den einmal aufgestellten Regeln gehorchen und somit deren ursprünglichen Sinn unterlaufen. Die Charaktere der Opern Gilberts und Sullivans qualifizieren sich somit als geistig eindimensional und demzufolge komisch.

$-7-$

Die unterschiedlichen moralischen Niveaus innerhalb der Gesellschaften Frankreichs und Englands in der zweiten Hälfte des 19. Jahrhunderts dürften darüber hinaus wesentlich die Gestalt der Stücke bestimmt haben. Hier war vor allem die über Jahrhunderte hinweg bewahrte Stabilität des Gesellschafts- und Sozialgefüges in England ausschlaggebend für die Konservierung einer biederen und puritanischen Geisteshaltung. Die gesellschaftlichen Verhältnisse in Frankreich hingegen waren weit weniger stabil. So erlebte Offenbach von seiner Ankunft in Paris bis zu seinem Tode vier verschiedene Regierungen und Regierungsformen, während Sullivan im Grunde genommen nur Königin Viktoria kannte. Diese unterschiedlichen politischen Voraussetzungen zeitigten nicht zuletzt Rückwirkungen auf das gesellschaftliche Leben, so dass Siegfried Kracauer nicht umhin konnte, die Zeit des Zweiten Kaiserreichs aufgrund materieller Prosperität und der Überbetonung sinnlicher Zerstreuung als "operettenhaft" zu analysieren. ${ }^{7}$ Da ein Erfolg der Stücke von der funktionierenden Interaktion zwischen Bühne und Publikum abhing, war es notwendig, dass die Zuschauer sich und das Gepräge ihres sozialen Umfeldes in den Stücken wiederfinden konnten. So verwundert es nicht, dass die Töchter General Stanleys in The Pirates of Penzance allenfalls ihre Schuhe ausziehen, um mit den Füßen im Wasser zu plätschern, während Offenbachs Librettisten Frivoles und Delikates unverhohlen an- und auch aussprechen.

\section{Das Sujet}

$-8-$

Mit dem Bezug zum Räubermilieu greifen beide Librettisten ein im 19. Jahrhundert allgemein äußerst beliebtes Themengebiet auf, welches zum einen in mehreren literarischen Werken seine Bearbeitung fand und zum anderen durch zahlreiche Sammlungen von Volks- und Räuberballaden eine Kodifizierung erfuhr. Ausgehend von dieser Popularität lag es somit nahe das Motiv 'Banditentum' früher oder später im Gattungsbereich der

7 Siegfried Kracauer: Jacques Offenbach und das Paris seiner Zeit, Frankfurt 1976, S. 186. 
Operette weiterzuentwickeln. Neben dem offensichtlichen Reiz des Verbrechens und des Schauers birgt dieses Sujet aber auch eine politische Pikanterie, denn in diesen Werken triumphieren anstatt der sonst üblichen Heldengestalten vor allem soziale Außenseiter und solche, die sich aus der bürgerlichen Gesellschaft zurückgezogen haben. Darüber hinaus lieferte gerade die ungeheure Popularität der Räuberthematik und die daraus zwangsläufig resultierende Abnutzung genügend Möglichkeiten für eine karikierende Bearbeitung, denn entgegen den zahlreichen Räuberopern der ersten Hälfte des 19. Jahrhunderts (z.B. Fra Diavolo, Ernani) wird in der Bearbeitung der Operette "das Räubermilieu, zum anderen die Räubergestalt in ihrem Zwiespalt zwischen dem grausamen Gewerbe und der das Mitgefühl des Publikums herausfordernden Menschlichkeit übertreibend und somit lächerlich dargestellt."

So haben es sich etwa die englischen Korsaren zum Prinzip gemacht, da sie selbst Waisen sind, auch keine anderen Waisen zu überfallen, und bleiben deshalb, da jeder davon weiß, chronisch erfolglos. Auch ihre französischen Kollegen jammern über desaströse Erträge, welche dadurch noch geschmälert werden, dass ihr Chef ständig neue Mitglieder in die Bande aufzunehmen bereit ist. Selbst die einstmals Schrecken und Gruseln hervorrufende Staffage ist auf ein Mindestmaß zurechtgestutzt. Gilberts Piraten müssen sich im I. Akt mit einer "Rocky Seashore on the Cost of Cornwall" begnügen, während der II. Akt sogar nur noch in einer "Ruined Chapel by Moonlight" beginnt. Auch Meilhac/Halévy reduzieren die Schauer auf das Nötigste. Ihre "Sauvagerie étrange" bleibt im Ungefähren, und allenfalls der Schatten eines Höhleneingangs vermag noch die Ahnung wilden Lebens zu erwecken. Somit bleibt die im Verlauf ihrer vielfachen literarischen Verarbeitung ohnehin schon ramponierte Räuberromantik nur Attrappe und das literarische Motiv des 'gerechten Räubers' erfährt eine erneute Vulgarisierung. ${ }^{9}$

$$
-10-
$$

Bei dem englischen Werk handelt es sich um ein burleskes Melodram. ${ }^{10}$ Dies gilt zum einen hinsichtlich der stereotypen Personenkonstellation,

$8 \quad$ Anna Amalie Abert: Räuber und Räubermilieu in der Oper des 19. Jahrhunderts (= Studien zur Musikgeschichte des 19. Jahrhunderts 42), hrsg. von Heinz Becker, Regensburg 1976, S. 123.

9 Elisabeth Frenzel: Räuber, der gerechte, in: Motive der Weltliteratur, Stuttgart ${ }^{3} 1988$, S. 599.

10 Hier im Sinne des in englischer wie französischer Literatur etablierten Schauerund Rührstücks mit unwahrscheinlicher Handlung, pathetischen Emotionen und einer Belohnung des Guten. 
wenn etwa in The Pirates of Penzance die Schurken (Piraten) unschuldige Mädchen (Generalstöchter) verfolgen, welche ein redlicher Held (Frederic) zu beschützen versucht, jedoch von einem Dummkopf (General Stanley) daran gehindert wird. Zum anderen weist aber auch die Entwicklung der Handlungslinie melodramatische Züge auf, indem die Geschichte geradewegs auf einen tragischen und gleichzeitig absurden Umschlag in Form eines coup de théâtre zuläuft. Nun müssen sich ungewöhnliche Charaktere aus für sie ungewöhnlichen Lagen befreien, was ihnen gelingt, indem sie prinzipientreu und vor allem logisch vorgehen und dabei für den Betrachter vollkommen plausibel handeln. Diese revolutionären Ideen oder frappierenden Entdeckungen (z.B. Frederic's Geburstag am 29. Februar) mit all den logischen Konsequenzen die sich daraus ableiten, markieren hier den komödiantischen Kulminationspunkt.

Im französischen Werk werden hingegen andere Merkmale akzentuiert. Hier sind vor allem das Intrigenspiel und leichtgewichtige Charaktere für die Handlung entscheidend, welche sich bei der Befriedigung ihrer Gelüste (Geld, Liebe, Vergnügen) von einer tragischen Situation in die nächste stoßen, denen sie entweder geistreich entfliehen oder solange scheitern, bis schließlich der Vorhang fällt. Am Ende entstehen hier von einer Situationskomik dominierte Stoffe, welche zwar anscheinend im Handlungsverlauf einer verborgenen inneren Logik folgen, in Wahrheit jedoch derartige Kausalität durch "reihende Wiederholung stereotyper Situationen"11 gewinnen. Der fortwährende Rekurs auf ein und dasselbe Handlungsschema kann dabei hinsichtlich seiner Intention durchaus ambivalent sein. So wäre etwa im Falle von Les Brigands durchaus zu fragen, ob Meilhac/Halévy mit dem dreimaligen Überfall der Räuber in wechselnder Verkleidung im II. Akt mit dem von ihnen gern verwandten Travestiemodell nicht vielmehr sich selbst parodieren.

Hinzu treten Unterschiede in der Handlungsführung. Während Gilbert die Entwicklung seiner Geschichte vornehmlich über Zufälle (coup de théâtre) vorantreibt, präferieren Halévy und Meilhac das Tableau, "denn im bürgerlichen Weltbild hat der Zufall - speziell in der willkürlich gewährten Begnadigung durch einen fürstlichen Deus ex machina — keinen Platz."12

11 Rainer Warning: Elemente einer Pragmasemiotik der Komödie, in: Das Komische (= Poetik und Hermeneutik 7), hrsg. von Rainer Warning und Werner Preisendanz, München 1976, S. 293. Gattung, Darmstadt 1998, S. 108. 
Die satirische Kritik zielt in beiden Werken auf das Militär und die Monarchie. In The Pirates of Penzance ist General Stanley ein vergreister Ordenträger, der sich mit einem gekauften Stammbaum Zugang zum Establishment verschaffen will und beim Anblick von Männern mit schwarzen Augenklappen unverzüglich weiche Knie bekommt. Zudem haben die Piraten einen König als Anführer, der es bezeichnenderweise ablehnt, ein standesgemäßes Leben zu führen. In Les Brigands repräsentieren mantuanische Höflinge und spanische Diplomaten das aristokratische Milieu, wobei letztere pikanterweise in "beaux hommes" sowie "hommes d'esprit" unterschieden werden. Auch hier macht die Herrschergestalt eine schlechte Figur. Der Hofstaat des Prinzen ist korrupt und er selber durch seine Begeisterung für das weibliche Geschlecht Mitauslöser einer horrenden Staatsverschuldung. Zudem entpuppen sich die Räuber als konvertierte Bankiers, die gehofft hatten, in ihrem neuen Beruf noch leichter an Geld heranzukommen, als das bisher ohnehin schon der Fall war. Daneben steht in beiden Libretti eine Gruppe von Polizisten als Widerpart, welche freilich unfähig ist, für Ordnung zu sorgen, da die einen immer zu spät kommen (Les Brigands), während den anderen ständig ihr Herz in die Stiefel rutscht (The Pirates of Penzance).

$-13-$

So sind es, da sich beide Librettisten den Luxus einer Nebenhandlung nicht leisten können, vor allem die Endpunkte der Handlungsentwürfe, die beide Werke voneinander unterscheiden. Abgesehen von der Tatsache, dass in Les Brigands die Räuber statt auf den Meeren im Wald ihr Unwesen treiben, kreist die Handlung um das die Laufbahn der Banditen krönende Schurkenstück. Unter Vorspielung falscher Tatsachen, nämlich der Verkleidung als spanische Gesandtschaft, soll am Hof von Mantua in Form einer Mitgift für die spanische Prinzessin eine millionenschwere Belohnung erschlichen werden. Hingegen ist die kriminelle Seite des Räuberlebens in The Pirates of Penzance zweitrangig. Zentral ist hier der Wunsch aller Beteiligten trotz widriger Umstände und ausgesprochen schlechter Reputation einen Ehepartner zu finden, was durch schicksalhafte Fügung und 'spirituelle' Mithilfe Königin Viktorias schließlich auch gelingt und in einer finalen Massenhochzeit vollzogen wird.

\section{Anmerkungen zum quantitativen Verhältnis von Dialog und Mu- sik}

$-14-$

Eine Betrachtung der Textumfänge zeigt ein deutlich größeres Textkorpus in Les Brigands gegenüber dem von The Pirates of Penzance. Dieses ist 
nicht nur durch die unterschiedliche Werkgliederung bedingt, sondern gilt auch absolut gesehen für jeden einzelnen Akt. Hierin könnte sich widerspiegeln, dass innerhalb der Entwicklung der französischen Oper von jeher ein stärkerer Bezug zum Sprechtheater mit besonderer Akzentuierung des Literarischen vorhanden war - bedingt allein schon durch den Gegensatz von durchkomponierter Grand Opéra zur Opéra comique mit gesprochenen Dialogen. ${ }^{13}$ Darüber hinaus zeigen die Dialoge in der englischen Version eine stärkere Tendenz zur Knappheit in Form von kurzen Aussagesätzen und tragen zudem häufig den Charakter spontanen, umgangsprachlichen Sprechens. Inwieweit hier eventuell auch ein höheres Sprechtempo des Französischen Auswirkungen auf die unterschiedlichen Textumfänge gehabt haben könnte, bleibt spekulativ. Der hohe Anteil gesprochener Abschnitte ließe sich generell als Unterscheidungsmerkmal zwischen Opéra bouffe beziehungsweise Savoy Opera ${ }^{14}$ und einer herkömmlichen Oper gebrauchen, da in letzterer das durchgängig begleitende Medium Musik Aufgaben übernehmen kann, die sonst der Text zu leisten hätte und er demzufolge dort sparsamer disponiert ist.

\section{$-15-$}

Bemerkenswert ist zudem, dass in beiden Werken der Textumfang je Akt im Verlauf kontinuierlich abnimmt. Hier ist zu vermuten, dass die wesentlichen Vorgänge und die entscheidende Handlungsverwicklung jeweils schon im I. Akt lokalisiert sind, was sich in der Übersicht der Handlungsverläufe auch zeigt. Im Falle von The Pirates of Penzance könnte jedoch der rückläufige Textumfang auch dadurch bedingt sein, dass hier im II. Akt der vokal dargebotene Teil des Librettos deutlich höher ist, und gesungene Passagen naturgemäß weniger Text enthalten als gesprochene. Der große Anteil gesprochener Passagen im letzten Akt von Les Brigands macht dabei zugleich deutlich, dass nicht immer ein ausgewogener Wechsel zwischen Musik und Dialog vorherrschen muss und mitunter mehrere dialogische Szenen aufeinander folgen können (Les Brigands III/Szenen 5-8).

$$
-16-
$$

Für beide Stücke lässt sich ein unterschiedlicher prozentualer Anteil gesprochenen Textes am gesamten Textkorpus konstatieren. Während in Les Brigands gesprochener Dialog über die Musikabschnitte dominiert, verhält es sich in The Pirates of Penzance genau umgekehrt. Hier treten die

13 Gabriele Buschmeier: Art. "Libretto", in: $M G G^{2}$ Sachteil Bd.5, Kassel 1996, Sp. 1155 . Theatre aufgeführten Werke. Später hingegen wurde er zum Synonym der Werke Gilbert und Sullivans. Vgl. dazu Meinhard Saremba: Arthur Sullivan. Ein Komponistenleben im viktorianischen England, Wilhelmshaven 1993, S. 289. 
Sprechszenen im II. Akt derart zurück, dass dieser letztendlich fast vollständig durchkomponiert erscheint. Vor diesem Hintergrund wäre Sullivans Werk als weitaus stärker musikalisiert zu kennzeichnen. Bei Offenbach hingegen besteht aufgrund der größeren epischen Anteile tendenziell eine größere Offenheit zum Schauspiel. Dieses wird nicht zuletzt auch dadurch bestätigt, dass Les Brigands das letzte Gemeinschaftswerk von Offenbach und seinen Librettisten Meilhac/Halévy darstellt, welche sich hernach verstärkt dem Sprechtheater zuwandten und mit Froufrou bereits im Jahr der Uraufführung von Les Brigands (1869) einen beachtlichen Erfolg erzielten. ${ }^{15}$ Fasst man die Erkenntnisse bezüglich der englischen Werke zusammen, so wäre bei einem typischen Werk Gilbert und Sullivans von einem zweiaktigen Opus mit Handlungshöhepunkt im I. Akt und annähernd ausgewogenem Verhältnis von Text und Musik zu sprechen. Dies bestätigt auch ein Blick auf alle 13 Savoy Opern und ist Ausdruck eines hohen Standardisierungsgrades innerhalb der künstlerischen Produktion. Bei Offenbachs Werken ist eine derartige Vereinheitlichung nicht zu erkennen. Hier scheint mit der formalen Gestaltung in weitaus stärkerem Maße experimentiert worden zu sein, da es in seinem Euvre neben dem hier betrachteten dreiaktigen Stück auch vier- und fünfaktige Werke mit noch verfeinerter Binnengliederung gibt. ${ }^{16}$ Zudem zeigt sein eigener Umarbeitungsdrang, dass sich Vorstellungen über Gestalt und Struktur einer Operette bei ihm im Laufe der Zeit wandelten ${ }^{17}$ und er im Gegensatz zu Sullivan, der im Bereich der komischen Oper fast ausschließlich mit Gilbert zusammenarbeitete, häufiger die Librettisten wechselte.

-17 -

Es bleibt einschränkend hinzuzufügen, dass die Textgestalt der hier besprochenen Werke bei weitem nicht so strikt determiniert gewesen sein dürfte wie die eines normalen Operntextes, denn mehr noch als dort lebte die Wirkung der Dialoge vor allem von aktualisierenden Extempores der Darsteller, die nur selten schriftlich fixiert waren. Darüber hinaus gibt es zahlreiche Hinweise in den Klavierauszügen, dass in Anpassung an die

Simone Monnier Clay: Henri Meilhac - Ludovic Halévy: Des bouffes-parisiens à l'Opéra-comique, Diss. Univ. of California, Ann Arbor 1987, S. 156. Vgl. etwa Geneviève de Brabant (1859), 2 Akte und 7 tableaux; 1867 umgearbeitet in 3 Akte und 9 tableaux.

So stellen etwa die beiden Fassungen von Le Pont des soupirs (1861/1868) trotz identischer Handlung nahezu verschiedene Werke dar. Während erstere noch ganz im Zeichen der absurden Hervé-Dramaturgie steht, zeigt die umgearbeitete Version Merkmale der 'klassischen' Opéra bouffe, was einerseits mit geändertem Publikumsgeschmack und andererseits dem von Offenbach 1864 vollzogenen Wechsel der Spielstätte (Théâtre des Variétés) in Verbindung zu bringen ist. 
jeweiligen Gegebenheiten auch ganze Passagen, wie etwa ein Melodram, wegfallen konnten (z.B. Les Brigands I/6a).

\section{Vergleich der Personenkonstellationen}

\section{Les Brigands}

Falsacappa, Chef du Brigands (T)

Fragoletto, Jeune fermier (T)

Pietro, Sous-Chef (T)

Antonio, Cassier du Duc du Mantoue (T)

Le Comte de Gloria-Cassis (T)

Le Baron de Campo-Tasso (T)

Le Duc de Mantoue (T)

Le Chef de Carabiniers (B)

Carmagnola, Brigand (T)

Domino (T) et Barbavano (B), Brigands

Pipo, Aubergiste (T)

Adolphe de Valladolid, Page (T)

Le Precepteur (B)

Fiorella, Fille du Bandit (S)

La Princesse de Grenade (S)

Zerlina, Fiametta, Bianca, Cicinella, Paysannes $(\mathrm{S})$

La Duchesse (S)

La Marquise (S)

Pipa, Femme de Pipo (S)

Pipetta, Fille de Pipo (S)

Isabel $(\mathrm{S})$

Brigands, Carabiniers, Paysannes, Marmitons, Pages de la cour de Mantoue, Seigneurs et dames de la cour de Grenade, Pages de la Princesse de Grenade

\section{The Pirates of Penzance}

The Pirate King (B)

Major-General Stanley (Bar)

Samuel, his Lieutenant (Bar)

Frederic, a Pirate Apprentice (T)

Sergeant of Police (B)

Mabel, General Stanley's daughter (S)

Ruth, a Pirate Maid-of-Work (Mez)

Edith (S)

Kate (Mez)

Pirates, Police and General Stanley's

Daughters

$-18-$

Die Übersicht der Personaltableaus zeigt auf der Seite von The Pirates of Penzance die für nahezu alle Werke Gilberts und Sullivans typische Rollenkombination: 
1. Ein Tenor (Frederic), der in einer Liebesbeziehung zu einer weiblichen Hauptrolle steht. Die stimmlichen Anforderungen (Spitzenton $g^{2}$ ) treten hinter schauspielerische wie Charme und Eleganz zurück.

2. Ein Sopran (Mabel) als romantische Liebespartnerin des Tenors mit welchem sie das Heldenpaar bildet. Dieser Part verlangt größere gesangstechnische Qualitäten, in diesem Falle Koloratursicherheit (Arie $\mathrm{I} / 8)$.

3. Ein lyrischer Bariton (General Stanley), der die Folie für den Komiker abgibt. Stimmlich ausschlaggebend sind gute Diktion (Pattersong I/13) und hohes Register (Notation im Sopranschlüssel).

4. Eine Alt- oder Mezzosopranstimme (Ruth) ${ }^{18}$ als Porträt älterer, zuweilen bizarrer Frauenpersönlichkeiten. Zusammen mit dem Bass (Pirate King) bildet sie als Gegenstück zu Frederic und Mabel das Buffopaar.

$$
-19-
$$

Hinzu treten eine Reihe kleinerer, unbedeutenderer Rollen (Samuel, Sergeant, Kate), die zumeist bestimmte Individuen der Chorgruppen repräsentieren und bei den Aufführungen wohl auch dementsprechend besetzt wurden. ${ }^{19}$ Die Chöre erhalten in beiden Werken eine nahezu selbständige Gruppenidentität. Bei Gilbert wird die Beziehung zu den Hauptcharakteren jedoch bewusst enger gehalten, wie beispielsweise im Falle Mabels durch ein Verwandtschaftsverhältnis, da deren Schwestern den Frauenchor bilden. Schließlich gibt es in Gilberts Konzeption keine reinen Sprechrollen wie in Les Brigands, da alle Handlungsträger auch am musikalischen Geschehen partizipieren. Ein Vergleich mit dem Personeninventar von Les Brigands lässt nur wenige Unterschiede in Bezug auf die Rollenverteilung deutlich werden:

1. Das Rollenangebot konzentriert sich im wesentlichen auf zwei Stimmlagen mit den dazugehörigen Fächern (lyrischer Tenor und Sopran, Soubrette und Spieltenor). Hingegen verzichtet Offenbach auf Altistin-

18 Jeffrey Leipsic ordnet in seinem Artikel The Pirates of Penzance die Rolle der Ruth zwar dem Sopranfach zu, doch sprechen Stimmumfang $\left(\mathrm{g}-\mathrm{e}^{2}\right)$ und Besetzungstraditionen eher dagegen, in: PEnz, Band 6, S. 174.

Andrew Hubbard: Musico-dramatic techniques in the late operas of Sullivan, Univ. Diss. Nottingham 1983, S. 171. 
nen und den für die Werke Gilberts und Sullivans charakteristischen Buffo-Bariton.

2. Bemerkenswert ist die Besetzung eines Contraltino-Parts (Antonio), der in seinem Couplet (III/20) hohe Diskantlagen $\left(\mathrm{d}^{2}\right) \mathrm{zu}$ bewältigen hat. Durch derartige stimmliche Übertreibungen wird die zur Schau gestellte männliche Virilität offenbar bewusst ins Lächerliche gezogen.

3. Ein Liebespaar bestehend aus Tenor und Sopran ist ebenfalls vorhanden. Hier jedoch kämpft die Soubrette auf der Seite der Räuber. Die Rolle des Polizeichefs ist in beiden Fällen eine Basspartie.

4. Resultierend aus der unterschiedlichen Anlage der Handlung ist das Personeninventar in Les Brigands insgesamt größer und mit zusätzlichen Nebenrollen (Dienern, Pagen), Chorgruppen (Hofstaat) und Statisterie angefüllt.

$$
-20-
$$

Des weiteren sehen Offenbach und seine Librettisten von einer komischen Frauenrolle ab. Ganz im Gegenteil ist Fiorella der Inbegriff emanzipierter Weiblichkeit, was sich nicht nur darin zeigt, dass ihrer Rolle zwei Solostücke zugestanden werden, sondern sich auch in der Art ihrer Deklamation und im musikalischen Ausdruck widerspiegelt, anhand dessen Offenbach sie als kecke Banditentochter porträtiert. Charakterrollen finden im Spektrum der hier betrachteten Werke keine Verwendung. Die dramatis personae werden von typisierten Figuren mit individuellem Gepräge gebildet, welches zwar charakteristische Züge haben kann, im wesentlichen aber aus dem sozialen und historischem Kontext heraus an Profil gewinnt. So mag General Stanley einerseits zwar auch eine Variation des in heiteren Bühnenwerken oft auftretenden miles gloriosus sein, die von ihm zur Schau getragene betuliche Haltung kennzeichnet ihn jedoch als unverwechselbares Mitglied seiner viktiorianischen Umgebung.

$$
-21-
$$

Beide Komponisten bevorzugen bei der Besetzung der einzelnen Rollen hauptsächlich hohe Stimmlagen. Die Gründe hierfür könnten einerseits im Vokalsatz liegen, der bei beiden Komponisten in den Ensembles hauptsächlich homophon ist, wobei die höchste Stimme automatisch melodietragend wird und somit Sopran und Tenor als exponierte Stimmen besser durchdringen. Andererseits dürften hohe Stimmen auch technisch eher dazu prädestiniert sein, die in den Stücken häufig vorkommenden Kadenzen und Koloraturen auszuführen. Darüber hinaus spielt die Kennzeichnung 
von Altersunterschieden eine wichtige Rolle. So muss etwa Ruth eine Mezzopartie sein, damit der Generationsunterschied zum mädchenhaften Sopran der Mabel deutlich wird. Genauso ist General Stanley ein Bariton, um ihn als alternden Vater klar vom jugendlichen Liebhaber Frederic abzugrenzen.

Trotz der Betonung einfachen und sinnlichen Amusements sind die stimmlichen Anforderungen an die einzelnen Partien vergleichsweise hoch. Dies zeigt sich nicht nur in den zahlreich vorhandenen Appogiaturen, sondern auch in den geforderten Stimmumfängen (z.B. Mabel: $c^{1}-c^{3}$ ). Da beide Werke aus einem fortgeschrittenen Stadium des künstlerischen Schaffens beider Komponisten stammen, stand ihnen offenbar zum Zeitpunkt der Entstehung mittlerweile ein exquisiteres Ensemble zur Verfügung. Darüber hinaus bleiben gesangliche Beweglichkeit (plappernde Achtelketten) und gewandte Spielbegabung für alle Partien wichtig.

$$
-23-
$$

Auffällig am Personeninventar ist für beide Werke die Aussparung des Proletariats, denn fast alle Figuren vertreten höhere Stufen einer ständischsozialen Ordnung. Offenbar war hier die Gefahr zu groß, mit tragischen und sozialkritischen Elementen eine Politisierung des Bühnengeschehens zu bewirken und somit den Unterhaltungswert der Stücke zu gefährden. Offenbach selbst bemerkte in seiner Beantragung eines Theaterprivilegs vom 24. 2. 1855, dass er vor allem beabsichtige, ein Genre zu etablieren, "qui serait de nature à plaire aux intelligences cultivés et à la masse des spectateurs. ${ }^{20}$ Festzuhalten ist auch, dass Hosenrollen (wie bei Offenbach die Figur Fragolettos) bei Gilbert und Sullivan fehlen. Offensichtlich scheuten die englischen Autoren vor derartigen Kostümspäßen mit Rücksicht auf die Befindlichkeiten des viktorianischen Publikums zurück. Offenbach und seinen Librettisten hingegen dürften die ästhetischen Reize, die mit dem Geschlechtertausch verbunden waren, sehr willkommen gewesen sein, bot sich hiermit doch einerseits die Möglichkeit, in den vielfach überlagerten Liebesbeziehungen zusätzliche komische Wirkungen zu erzielen, und andererseits die Gelegenheit, das jugendliche Alter einer Person wie Fragoletto zu kennzeichnen, der bei seinem ersten Auftritt als "très petit homme" vorgestellt wird.

20 Nicole Wild: Dictionnaire des théatres parisiens aux XIX $X^{e}$ siècle. Les théatres et la musique, Paris 1989, S.61. 


\section{Analyse der Handlungsverläufe}

\section{Les Brigands}

I. Akt (1743)*

\begin{tabular}{|c|c|c|c|c|c|}
\hline Nr. & |Personen & Inhalt & Form & Tonart & $\begin{array}{l}\begin{array}{l}\text { Tempo }-\mathrm{Me}- \\
\text { trum }\end{array} \\
\end{array}$ \\
\hline 0 & - & & Ouvertüre & $\begin{array}{l}\text { A-Dur (mo- } \\
\text { dulierend) }\end{array}$ & $\begin{array}{l}\text { Allegro mode- } \\
\text { rato }-2 / 4\end{array}$ \\
\hline $1 \mathrm{a}$ & Räuber & $\begin{array}{l}\text { Räuber patroullie- } \\
\text { ren }\end{array}$ & Eingangschor & G-Dur & Moderato - C \\
\hline $1 b$ & $\begin{array}{l}\text { junge Mädchen, } \\
\text { Falsacappa }\end{array}$ & $\begin{array}{l}\text { Falsacappa entführt } \\
\text { Mädchen }\end{array}$ & Couplet & G-Dur & Allegretto $-2 / 4$ \\
\hline $1 \mathrm{c}$ & Falsacappa & $\begin{array}{l}\text { Der Bandenchef } \\
\text { stellt sich vor }\end{array}$ & Couplet & As-Dur & Allegro $-6 / 8$ \\
\hline $1 d$ & $\begin{array}{l}\text { Räuber, junge } \\
\text { Mädchen }\end{array}$ & $\begin{array}{l}\text { Huldigung Falsa- } \\
\text { cappas }\end{array}$ & $\begin{array}{l}\text { Ensemble } \\
\text { (Stretta) }\end{array}$ & G-Dur & $\begin{array}{l}\text { Allegro/Presto } \\
-3 / 8\end{array}$ \\
\hline 1bis & $\begin{array}{l}\text { Räuber, Falsa- } \\
\text { cappa, Fiorella }\end{array}$ & $\begin{array}{l}\text { Falsacappa ver- } \\
\text { spricht großen } \\
\text { Coup }\end{array}$ & Melodram & $\begin{array}{l}\text { As-Dur/Des- } \\
\text { Dur }\end{array}$ & $3 / 4$ \\
\hline 2 & Fiorella & $\begin{array}{l}\text { Die Räubertochter } \\
\text { stellt sich vor }\end{array}$ & Couplet & As-Dur & $3 / 4$ \\
\hline 3 & Tutti & $\begin{array}{l}\text { Gefangennahme } \\
\text { Fragolettos }\end{array}$ & Ensemble & $\begin{array}{l}\text { B-Dur (mo- } \\
\text { dulierend) }\end{array}$ & $\begin{array}{l}\text { Allegro vivo - } \\
2 / 4\end{array}$ \\
\hline 4 & Fragoletto & $\begin{array}{l}\text { Fragoletto möchte } \\
\text { Räuber werden }\end{array}$ & Couplet & As-Dur & Allegro - 3/4 \\
\hline 4bis & Räuber & & $\begin{array}{l}\text { Abgangsscho } \\
\mathrm{r}\end{array}$ & B-Dur & $\begin{array}{l}\text { Allgro vivo - } \\
2 / 4\end{array}$ \\
\hline 5 & Fiorella, Prinz & $\begin{array}{l}\text { Fiorella weist ihm } \\
\text { den Weg }\end{array}$ & $\begin{array}{l}\text { Rondoduett } \\
\text { (ABA'CA") }\end{array}$ & F-Dur & $\begin{array}{l}\text { Allegro mode- } \\
\text { rato }-2 / 4\end{array}$ \\
\hline 6 & $\begin{array}{l}\text { Räuber, Frago- } \\
\text { letto }\end{array}$ & $\begin{array}{l}\text { Fragoletto nimmt } \\
\text { Kurier gefangen }\end{array}$ & $\begin{array}{l}\text { Saltarello } \\
\text { (Solo + Chor) }\end{array}$ & Es-Dur & Presto $-6 / 8$ \\
\hline 7 & Tutti & $\begin{array}{l}\text { Aufnahme Frago- } \\
\text { lettos und Orgie }\end{array}$ & $\begin{array}{l}\text { Finalensembl } \\
\mathrm{e}\end{array}$ & $\begin{array}{l}\text { D-Dur (mo- } \\
\text { dulierend) }\end{array}$ & $\begin{array}{l}\text { Beginn: Mode- } \\
\text { rato - C }\end{array}$ \\
\hline II. Akt & (1407) & & & & \\
\hline 8 & - & & Entr'acte & A-Dur & Allegro $-3 / 8$ \\
\hline 9 & Herbergsleute & $\begin{array}{l}\text { Vorbereitung für } \\
\text { spanische Gäste }\end{array}$ & Eingangschor & E-Dur & $\begin{array}{l}\text { Allegro vivo - } \\
2 / 4\end{array}$ \\
\hline 9 bis & Herbergsleute & & Abgangschor & E-Dur & $\begin{array}{l}\text { Allegro vivo - } \\
2 / 4\end{array}$ \\
\hline 10 & $\begin{array}{l}\text { Räuber, Bäue- } \\
\text { rinnen }\end{array}$ & $\begin{array}{l}\text { Räuber begehren } \\
\text { Einlass }\end{array}$ & $\begin{array}{l}\text { Ensemble } \\
\text { (Kanon ) }\end{array}$ & G-Dur & Moderato - C \\
\hline 11 & $\begin{array}{l}\text { Fiorella, Frago- } \\
\text { letto, Räuber }\end{array}$ & $\begin{array}{l}\text { Fiorella will Fra- } \\
\text { goletto heiraten }\end{array}$ & Duett & $\begin{array}{l}\text { As-Dur (mo- } \\
\text { dulierend) }\end{array}$ & Allegretto $-2 / 4$ \\
\hline $11 \mathrm{bis}$ & Tutti & $\begin{array}{l}\text { Räuber besingen } \\
\text { ihren Wagemut }\end{array}$ & Abgangschor & As-Dur & Allegro - 2/4 \\
\hline
\end{tabular}




\begin{tabular}{|c|c|c|c|c|c|}
\hline 12 & $\begin{array}{l}\text { Fragoletto, Fal- } \\
\text { sacappa, Pietro }\end{array}$ & $\begin{array}{l}\text { Falsacappa erklärt } \\
\text { seinen Plan }\end{array}$ & $\begin{array}{l}\text { Terzett } \\
\text { (dialogisieren } \\
\text { d) }\end{array}$ & $\begin{array}{l}\text { As-Dur/D- } \\
\text { Dur }\end{array}$ & $\begin{array}{l}\text { Allegro/ Mode- } \\
\text { rato - 6/8, 3/4 }\end{array}$ \\
\hline 13 & Räuber & $\begin{array}{l}\text { Räuber vollständig } \\
\text { verkleidet }\end{array}$ & $\begin{array}{l}\text { Chor und } \\
\text { Melodram }\end{array}$ & B-Dur & $\begin{array}{l}\text { Maestoso - C, } \\
\text { 2/4 }\end{array}$ \\
\hline $14 \mathrm{a}$ & $\begin{array}{l}\text { Räuber, spani- } \\
\text { sche Gesandte }\end{array}$ & $\begin{array}{l}\text { Botschafter erkun- } \\
\text { den die Herberge }\end{array}$ & $\begin{array}{l}\text { Ensemble - } \\
\text { Couplet }\end{array}$ & $\begin{array}{l}\text { F-Dur/As- } \\
\text { Dur }\end{array}$ & $\begin{array}{l}\text { Moderato-Alle- } \\
\text { gretto }-6 / 8,2 / 4\end{array}$ \\
\hline $15 \mathrm{a}$ & $\begin{array}{l}\text { Räuber, spani- } \\
\text { sche Gesandte }\end{array}$ & $\begin{array}{l}\text { Räuber zerren Spa- } \\
\text { nier ins Haus }\end{array}$ & Chor & a-moll & $\begin{array}{l}\text { Allegro vivo - } \\
2 / 4\end{array}$ \\
\hline $15 b$ & $\begin{array}{l}\text { spanischer } \\
\text { Hofstaat }\end{array}$ & $\begin{array}{l}\text { Einzug der spani- } \\
\text { schen Prinzessin }\end{array}$ & $\begin{array}{l}\text { Melodram - } \\
\text { Szene }\end{array}$ & E-Dur & Moderato $-3 / 4$ \\
\hline $15 \mathrm{c}$ & Gloria-Cassis & $\begin{array}{l}\text { Kammerherr be- } \\
\text { singt Nationalstolz }\end{array}$ & Couplet & A-Dur & Allegro $-3 / 8$ \\
\hline 16 & $\begin{array}{l}\text { Fiorella, Frago- } \\
\text { letto, Prinzessin, } \\
\text { Adolphe }\end{array}$ & $\begin{array}{l}\text { Fiorella erklärt ihre } \\
\text { Liebe gegenüber } \\
\text { Fragoletto }\end{array}$ & Couplet & F-Dur & $\begin{array}{l}\text { Allegretto mode- } \\
\text { rato }-6 / 8\end{array}$ \\
\hline $17 \mathrm{a}$ & Räuber & $\begin{array}{l}\text { Räuber bitten Prin- } \\
\text { zessin ins Haus }\end{array}$ & Chor & a-moll & $\begin{array}{l}\text { Allegro vivo - } \\
2 / 4\end{array}$ \\
\hline $17 b$ & Räuber & $\begin{array}{l}\text { Vorfreude auf die } \\
\text { Millionenbeute }\end{array}$ & Ensemble & A-Dur & $\begin{array}{l}\text { Allegro marziale } \\
-2 / 4\end{array}$ \\
\hline $17 \mathrm{c}$ & $\begin{array}{l}\text { Räuber, spani- } \\
\text { scher Hofstaat }\end{array}$ & $\begin{array}{l}\text { Spanier entdecken } \\
\text { die Hinterlist }\end{array}$ & Szene & $\begin{array}{l}\text { e-moll/g- } \\
\text { moll }\end{array}$ & $\begin{array}{l}\text { Allegro/Allegrett } \\
0-6 / 8, C\end{array}$ \\
\hline $17 d$ & $\begin{array}{l}\text { Räuber, Spanier, } \\
\text { Carabiniers }\end{array}$ & $\begin{array}{l}\text { Räuber nehmen } \\
\text { Spanier und Cara- } \\
\text { biniers gefangen }\end{array}$ & Strett & $\begin{array}{l}\text { A-Dur (mo- } \\
\text { dulierend) }\end{array}$ & Allegretto $-2 / 4$ \\
\hline $\begin{array}{l}\text { III. } \\
\text { Akt }\end{array}$ & (745) & & & & \\
\hline 18 & & & Entr'acte & E-Dur & Allegro $-2 / 4$ \\
\hline $19 a$ & $\begin{array}{l}\text { Damen des Ho- } \\
\text { fes von Mantua }\end{array}$ & $\begin{array}{l}\text { Trauer wegen Hei- } \\
\text { rat des Herzogs }\end{array}$ & Cho & E-Dur & $\begin{array}{l}\text { Très modéré - } \\
6 / 8\end{array}$ \\
\hline $19 b$ & $\begin{array}{l}\text { Herzog von } \\
\text { Mantua }\end{array}$ & $\begin{array}{l}\text { Junggesellenab- } \\
\text { schied des Herzogs }\end{array}$ & Couplet & G-Dur & $\begin{array}{l}\text { Allegro mode- } \\
\text { rato }-2 / 4\end{array}$ \\
\hline 20 & $\begin{array}{l}\text { Kassierer des } \\
\text { Herzogs }\end{array}$ & $\begin{array}{l}\text { Kummer wegen } \\
\text { leerer Staatskasse }\end{array}$ & Couplet & B-Dur & Andante $-2 / 4$ \\
\hline 21 & $\begin{array}{l}\text { Hofstaat von } \\
\text { Mantua, Räuber }\end{array}$ & $\begin{array}{l}\text { Ankunft der Räuber } \\
\text { am Hof }\end{array}$ & Ensemble & $\begin{array}{l}\text { B-Dur (mo- } \\
\text { dulierend) }\end{array}$ & Allegro - 3/8 \\
\hline bis & $\begin{array}{l}\text { Hofstaat von } \\
\text { Mantua, Räuber }\end{array}$ & & Abgangschor & Es-Dur & Allegro - 2/4 \\
\hline 22 & Tutti & $\begin{array}{l}\text { Aufdeckung des } \\
\text { Schwindels }\end{array}$ & $\begin{array}{l}\text { Finalensembl } \\
\mathrm{e}\end{array}$ & $\left|\begin{array}{l}\text { a-moll (mo- } \\
\text { dulierend) }\end{array}\right|$ & $\begin{array}{l}\text { Beginn: Vivo - } \\
6 / 8\end{array}$ \\
\hline
\end{tabular}

* Die Ziffern in Klammern geben die Taktanzahl je Akt an 
The Pirates of Penzance or The Slave of Duty

I. Akt (1640)

\begin{tabular}{|c|c|c|c|c|c|}
\hline Nr. & Personen & Inhalt & Form & Tonart & Tempo-Metrum \\
\hline 0 & & & Ouvertüre & $\begin{array}{l}\text { C-Dur (mo- } \\
\text { dulierend) }\end{array}$ & $\begin{array}{l}\text { Allegro maestoso } \\
\text { - alla breve }\end{array}$ \\
\hline 1 & Piraten, Samuel & $\begin{array}{l}\text { Piraten feiern Fre- } \\
\text { derics Geburtstag }\end{array}$ & $\begin{array}{l}\text { Eingangs- } \\
\text { chor }\end{array}$ & Es-Dur & $\begin{array}{l}\text { Moderato maestoso } \\
-6 / 8\end{array}$ \\
\hline 2 & Ruth & $\begin{array}{l}\text { Ruth gesteht ihre } \\
\text { Schwerhörigkeit }\end{array}$ & Song & c-moll & $\begin{array}{l}\text { Allegro pesante - } \\
2 / 4\end{array}$ \\
\hline 3 & $\begin{array}{l}\text { Piratenkönig, Pi- } \\
\text { raten }\end{array}$ & $\begin{array}{l}\text { Glorifizierung des } \\
\text { Piratenlebens }\end{array}$ & Song & C-Dur & $\begin{array}{l}\text { Allegro moderato } \\
-6 / 8\end{array}$ \\
\hline 4 & Frederic, Ruth & $\begin{array}{l}\text { Frederic verstößt } \\
\text { Ruth }\end{array}$ & $\begin{array}{l}\text { Rezitativ } \\
\text { und Duett }\end{array}$ & $\begin{array}{l}\text { g-moll/G- } \\
\text { Dur }\end{array}$ & $\begin{array}{l}\text { Allegro vivace/An- } \\
\text { dante }-\mathrm{C}, 6 / 8\end{array}$ \\
\hline 5 & Generalstöchter & $\begin{array}{l}\text { Ausflug der Töch- } \\
\text { ter zur Küste }\end{array}$ & $\begin{array}{l}\text { Auftrittsch } \\
\text { or }\end{array}$ & $\begin{array}{l}\text { Es-Dur/B- } \\
\text { Dur }\end{array}$ & $\begin{array}{l}\text { Allegro grazioso } \\
-2 / 4\end{array}$ \\
\hline 6 & $\begin{array}{l}\text { Frederic, General- } \\
\text { stöchter }\end{array}$ & $\begin{array}{l}\text { Frederic bittet um } \\
\text { Zuneigung }\end{array}$ & $\begin{array}{l}\text { Rezitativ } \\
\text { - Arioso }\end{array}$ & $\begin{array}{l}\text { B-Dur (mo- } \\
\text { dulierend) }\end{array}$ & $\begin{array}{l}\text { Andante moderato } \\
-\mathrm{C}\end{array}$ \\
\hline 7 & $\begin{array}{l}\text { Frederic, Mabel, } \\
\text { Töchter }\end{array}$ & $\begin{array}{l}\text { Töchter haben } \\
\text { Angst vor Piraten }\end{array}$ & $\begin{array}{l}\text { dialogisie- } \\
\text { rendes } \\
\text { Ensemble }\end{array}$ & Des-Dur & Andante $-3 / 4$ \\
\hline 8 & $\begin{array}{l}\text { Mabel, General- } \\
\text { stöchter }\end{array}$ & $\begin{array}{l}\text { Frederics Flehen } \\
\text { wird erhört }\end{array}$ & $\begin{array}{l}\text { Da-capo- } \\
\text { Arie ABA' }\end{array}$ & As-Dur & $\begin{array}{l}\text { Modo di valse - } \\
3 / 4\end{array}$ \\
\hline 9 & Generalstöchter & $\begin{array}{l}\text { Töchter sprechen } \\
\text { über das Wetter }\end{array}$ & $\begin{array}{l}\text { dialogisie- } \\
\text { rendes } \\
\text { Ensemble }\end{array}$ & As-Dur & Allegretto $-\mathrm{C}$ \\
\hline 10 & $\begin{array}{l}\text { Frederic, Mabel, } \\
\text { Töchter }\end{array}$ & Liebesbekenntnis & $\begin{array}{l}\text { Duett und } \\
\text { Chor }\end{array}$ & $\begin{array}{l}\text { H-Dur/G- } \\
\text { Dur }\end{array}$ & $\begin{array}{l}\text { Allegro vivace - } \\
2 / 4\end{array}$ \\
\hline 11 & $\begin{array}{l}\text { Frederic, Piraten, } \\
\text { Töchter }\end{array}$ & $\begin{array}{l}\text { Piraten fallen ein } \\
\text { und wollen die } \\
\text { Töchter heiraten }\end{array}$ & $\begin{array}{l}\text { Rezitativ } \\
\text { und Chor }\end{array}$ & $\begin{array}{l}\text { H-Dur/G- } \\
\text { Dur }\end{array}$ & $\begin{array}{l}\text { Allegretto/Vivace } \\
-3 / 2, \text { alla breve }\end{array}$ \\
\hline 12 & $\begin{array}{l}\text { Mabel, General, } \\
\text { Töchter, Piraten }\end{array}$ & $\begin{array}{l}\text { Töchter verweisen } \\
\text { auf ihren mächti- } \\
\text { gen Vater }\end{array}$ & $\begin{array}{l}\text { Rezitativ } \\
\text { und Chor }\end{array}$ & $\begin{array}{l}\text { g-moll/B- } \\
\text { Dur }\end{array}$ & Moderato $-\mathrm{C}, 6 / 8$ \\
\hline 13 & $\begin{array}{l}\text { General, Töchter, } \\
\text { Piraten }\end{array}$ & $\begin{array}{l}\text { General Stanley } \\
\text { stellt sich vor }\end{array}$ & Couplet & Es-Dur & $\begin{array}{l}\text { Allegro vivace - } \\
\text { alla breve }\end{array}$ \\
\hline 14 & Tutti & $\begin{array}{l}\text { Piraten lassen } \\
\text { Gnade walten, da } \\
\text { der General angeb- } \\
\text { lich Waise ist }\end{array}$ & $\begin{array}{l}\text { mehrteili- } \\
\text { ges Final- } \\
\text { ensemble }\end{array}$ & $\begin{array}{l}\text { g-moll/D- } \\
\text { Dur/F-Dur } \\
\text { G-Dur }\end{array}$ & $\begin{array}{l}\text { Beginn: Moderato } \\
\text { C, } 6 / 8\end{array}$ \\
\hline II. Akt & (1121) & & & & \\
\hline 15 & Mabel, Töchter & $\begin{array}{l}\text { Töchter trösten } \\
\text { ihren Vater }\end{array}$ & $\begin{array}{l}\text { Eingangs- } \\
\text { chor }\end{array}$ & Es-Dur & $\begin{array}{l}\text { Allegro con tene- } \\
\text { rezza }-6 / 8\end{array}$ \\
\hline 16 & $\begin{array}{l}\text { General Stanley, } \\
\text { Frederic }\end{array}$ & $\begin{array}{l}\text { General hofft auf } \\
\text { rettende Polizei }\end{array}$ & Rezitativ & C-Dur & Moderato - C \\
\hline
\end{tabular}




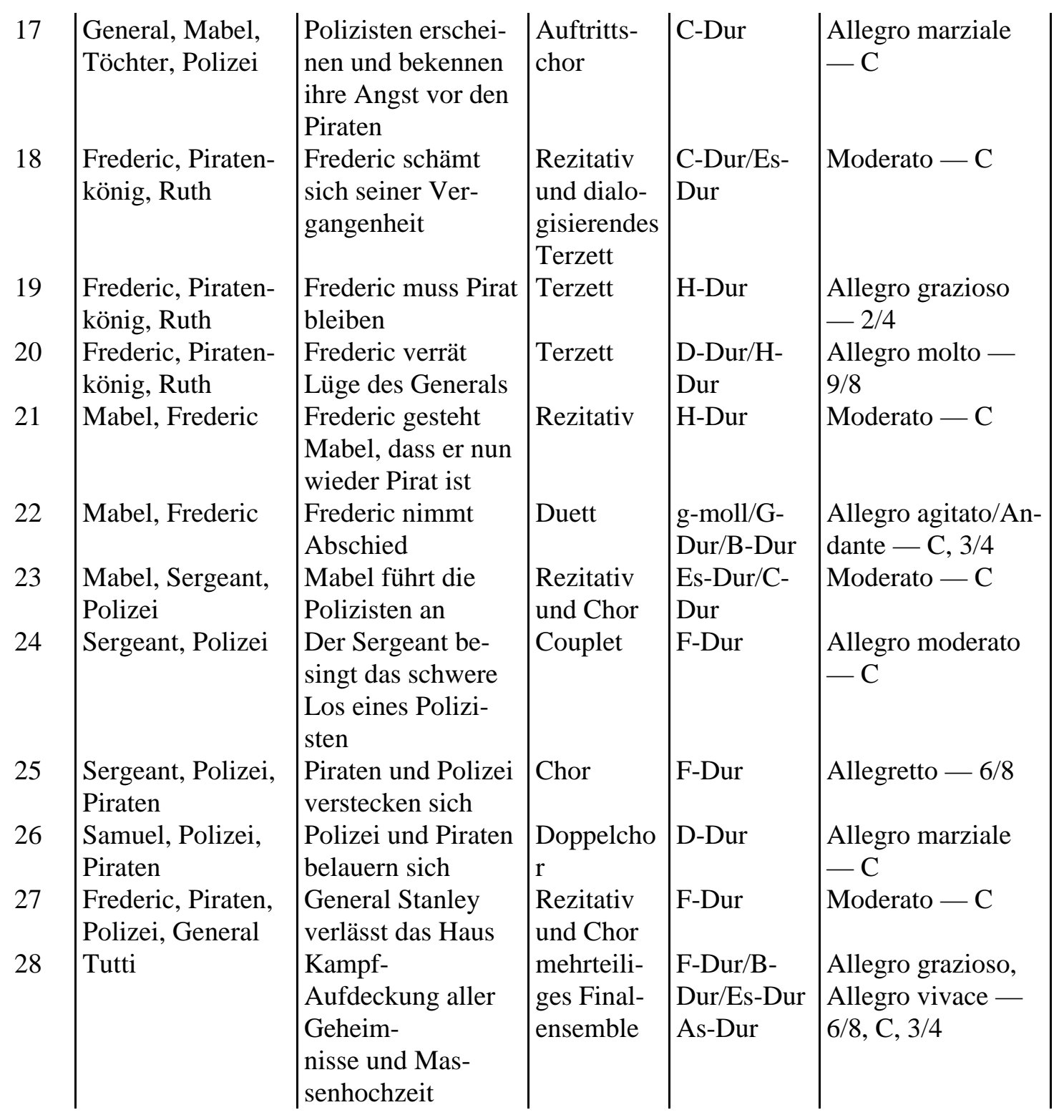

Die Gegenüberstellung der Handlungsverläufe macht deutlich, dass beide Komponisten ihre Werke dramaturgisch ähnlich ausrichten. Die Stücke werden zunächst von einer Ouvertüre eingeleitet, welche zwar eine erste atmospärische Schilderung bietet, hinsichtlich ihres musikalischen Gehalts jedoch kaum interessant ist. Thematische Entwicklung findet, wenn überhaupt, nur rudimentär statt, und in der Musik klingen die zu erwartenden Zugnummern kurz an. Obgleich Michael Klügl ${ }^{21}$ für einige Ouvertüren Offenbachs die Funktion eines dramaturgischen Inhaltsverzeichnisses glaubhaft belegt, besitzen die hier untersuchten Beispiele in beiden Fällen kaum

21 Michael Klügl: Erfolgsnummern. Modelle einer Dramaturgie der Operette (= Thurnauer Schriften zum Musiktheater 13), Laaber 1992. 
mehr als Potpourri-Charakter. ${ }^{22}$ Beide Komponisten verbindet die Gepflogenheit, das Stück im Anschluss an die Ouvertüre mit einem Chor einzuleiten, welcher eindeutig koloritstiftende Funktion besitzt. So wird neben der Vermittlung von Zeit, Ort und Raum des Geschehens, das Publikum auch atmosphärisch auf den Theaterabend eingestimmt. Allerdings wählt Offenbach im Gegensatz zu Sullivan nicht ein herkömmliches Chorensemble, sondern das Modell einer ausgebauten Chor-Introduktion und greift damit auf eine in der Opéra comique der ersten Hälfte des 19. Jahrhunderts etablierte Konvention der Werkeröffnung zurück. Dieses Modell hatte sich dort vor allem deshalb bewährt, "weil es die Expositionsfunktionen der deskriptiven Situationsschilderung mit der narrativen Informationsvergabe in harmonischer Weise zu verbinden vermochte."23

\section{$-25-$}

Im Anschluss daran kommt es zu einem regelmäßigen Wechsel von gesungenen, gesprochenen und instrumentalen Abschnitten, was ebenfalls ein Erbe der Opéra comique beziehungsweise des Singspiels sein dürfte. Dabei sind die Dialoge durchgängig in Prosa, die Gesangstexte stets in Versen abgefasst. Treten gereimte Dialoge auf, so ist damit zumeist eine besondere Ausdrucksqualität verbunden, wenn etwa Falsacappa dem Herzog von Mantua höflich eröffnet, dass sein Finanzminister ein Dieb ist (III/10). Da ein Großteil der Musiknummern statischen Charakter trägt und hauptsächlich kommentierende Funktionen besitzt, vollzieht sich die eigentliche Handlung im Dialog. Hier wird zumeist ein Problem aufgeworfen oder ein Affekt entwickelt, bevor sich die Spannung dann in der anschließenden Musik entlädt. Die Nummerndramaturgie wird offenbar von der Oper übernommen, ohne gleichzeitig deren festgefügte Szenenabfolge mit zu adaptieren, welche beide Komponisten durch einen Verbund von Einlagestücken ersetzen. Zusammen mit der strikten Trennung von Gesang und Dialog wurde dadurch eine variable Handlungsführung möglich, wobei insbesondere durch schnellere Szenenwechsel Monotonie vermieden wer-

Roger Harris belegt anhand von Tagebucheinträgen, dass mindestens die Hälfte der Ouvertüren zu den Opern Sullivans nicht von ihm selbst stammen, sondern von Assistenten, nicht selten noch in der Nacht vor der Premiere, aus markanten Motiven und Passagen der jeweiligen Werke zusammengeschrieben wurden, in: Gilbert and Sullivan. Papers presented at the International Conference held at the University of Kansas, hrsg. von James Helyar, Kansas 1970, S. 75. Die Opéra comique und ihr Einfluß auf das europäische Musiktheater im 19. Jahrhundert. Bericht über den internationalen Kongreß Frankfurt 1994 (= Musikwissenschafliche Publikationen 3), hrsg. von Herbert Schneider und Nicole Wild, Hildesheim 1997, S. 167. 
den konnte. Der erste Akt ist in beiden Werken expositorisch aufgebaut. Er leistet zunächst das Kennenlernen des Liebespaares, was sich bei Sullivan im ersten Drittel des I. Akts vollzieht (I/10), wogegen es bei Offenbach erst im Finale musikalisch zueinander findet. Hinzu kommen die Entrées der Protagonisten und die obligatorische Buffonummer (Offenbach: I/6; Sullivan: I/13). Hinsichtlich der Nummernfolge scheint als halbwegs beachtete Maxime gegolten zu haben, eine möglichst abwechslungsreiche Dramaturgie zu bieten, indem Solo- und Ensembleformationen einander annähernd regelmäßig abwechseln. Auffallend ist, dass Sullivan im Gegensatz zu Offenbach am Beginn des I. Akts noch streng nach Frauen- und Männerchören trennt und sie erst zum Aktschluss zusammenführt.

\section{$-26-$}

Unterschiedlich ist im weiteren szenischen Ablauf die Positionierung der Chöre. Hier ist bei Offenbach die Eigenart zu erkennen, einzelne Musiknummern durch gesprochene Abschnitte zu durchbrechen, an welche sich dann ein Abgangschor anschließt (I/4, II/9, III/21), der besonders markante Devisen oder den Refrain eines Couplets rekapituliert. In The Pirates of Penzance gibt es das nicht. Hier werden Musik und gesprochene Passagen entweder klar voneinander getrennt oder aber kurze Textabschnitte in Form von mehr oder weniger freien Rezitativen in ein Ensemble eingebunden. Ebenso fehlen im englischen Werk melodramatische Abschnitte. Mit diesen knüpfte Offenbach an die französische Operntradition an, wo das Melodram in der Opéra-comique einen festen Platz hatte und in den 1850er Jahren einen großen Aufschwung sowohl im Musiktheater als auch in anderen Gattungen nahm (s. Schumanns Melodramen). Sullivan hingegen greift in diesem Bereich reglementierter Deklamation generell auf die Form des Rezitativs zurück, um den Übergang zwischen gesprochenen und gesungenen Passagen abzustufen. Sehr oft stehen dabei parodistische Aspekte im Vordergrund, wenn etwa das in der romantischen Oper schon zum Stereotyp erstarrte alte Nummernprinzip aus Szene und Arie bewusst zitiert wird, oder aber sämtliche stilistischen wie musikalisch-rhetorischen Klischees der Rezitative barocker Oratorien zusammengezogen werden (so zum Beispiel Rezitativ des Frederic II/18). Der ausdrückliche Bezug Offenbachs zur zeitgenössischen Theaterpraxis spiegelt sich auch in den Zwischenaktmusiken wider, mit denen er jeden Aufzug einleitet. Neben einer denkbaren Funktion als Kaschierung einer durch etwaige Bühnenumbauten enstehenden Geräuschkulisse, werden hier für Interpreten wie Publikum die wesentlichen Parameter des zu erwartenden Aktverlaufs knapp umrissen. Sullivan hingegen bündelt diese musikalische Informationsvergabe einzig in der einleitenden Ouvertüre. 
Überblickt man die Terminologie der einzelnen Musiknummern, so wird bei Offenbach die Einflussnahme der Tanzmusik auf die Operettenproduktion sehr deutlich. Neben einigen explizit als Tanz ausgewiesenen Abschnitten (z.B. I/6 Saltarello, I/7 Tarantella) ist in einer Vielzahl der Musiknummern die musikalische Faktur aus Tanzidiomen abgeleitet. Für Offenbach dürften die Tänze vor allem durch ihren motorischen Impetus und hinsichtlich ihrer rhythmischen Prägnanz interessant gewesen sein, "welche nicht nur die Zuspitzung des musikalischen Geschehens in Form der üblichen Stretta erlaubte, sondern darüber hinaus auch die Übersetzung der musikalischen Ereignisse in körperliche Bewegung" ${ }^{24}$ möglich machte. Die Annäherung an populärmusikalische Strukturen war aber auch dem Bemühen um eine konzisere Ausgestaltung der Couleur locale geschuldet, denn mit dem Räuberleben waren vor allem Chiffren wie Sinnesfreude und Lebenslust konnotiert, als deren angemessener Ausdruck Tänze gemeinhin gelten konnten. Sullivan hingegen setzt Tänze nur sehr sparsam und weniger unter motorischen Gesichtspunkten ein. Vielmehr wird auf deren Symbolwert Bezug genommen, wie etwa im parodistischen Rekurs auf Gesangsmodelle der großen romantischen Oper durch Mabels Koloraturwalzer $(\mathrm{I} / 8)$.

$$
-28-
$$

Beim Vergleich der Binnengliederung der einzelnen Akte zeigt sich in The Pirates of Penzance ein symmetrischer Bau. Mit je 14 Nummern stehen hier im Gegensatz zu Les Brigands gleichviele Musikabschnitte zur Verfügung. Dieses liegt vornehmlich daran, dass Offenbach die Musik zeitweilig, etwa in der anfänglichen Introduktion oder in den beachtlich proportionierten Aktschlüssen, in größeren szenisch-musikalischen Komplexen zusammenfasst, wohingegen Gilbert und Sullivan konsequent nach dem Einlageprinzip verfahren und einzelne kleinere Musiknummern in ihrer Eigenständigkeit erhalten, weshalb die zeitgenössische Kritik nicht müde wurde anzumerken, ihre Werke litten aufgrund der vielteiligen Einlagenstruktur an szenischer Kurzatmigkeit. ${ }^{25}$ Handbuch der musikalischen Gattungen Bd. 13), Laaber 1997, S. 301.

"That Dr. Sullivan has left Mr. Gilbert behind him wherever the setting is most striking will hardly be denied, for the setting is striking in passages although somewhat difficult to reconcile with the unities. Here a graceful love passage, here a lively patter song, here an impressive chant, here a pathetic prayer, present the varied charms of melody, energy, or religious sentiment. If at times we are bewildered as to whether we are at the Oxford or at Exeter Hall, if we are in doubt as to whether the next number will be in the style of negro minstrelsy or an oratorio, 
Bei beiden Komponisten ist die Vorliebe für einfache und leicht fassliche Liedformen zu beobachten. Hier scheint mit der dominierenden Form des Couplets (bzw. bei Sullivan: Song) ein angemessener Ausdruck für die gewünschte Vereinfachung und Popularisierung der Gattung gefunden worden zu sein, denn diese Stücke dienten offenbar nicht der Entwicklung der Handlung beziehungsweise der Vermittlung empfindsamer Momente, sondern exponierten neben einer bestimmten Figur vor allem nachvollziehbares und reproduzierbares Musikmaterial. ${ }^{26}$ Dies umso mehr, als die einzelnen Personen in ihrer Entwicklung innerhalb der Operette zumeist keiner Steigerung im dramatischen Kontext unterliegen und die Solonummern auch gleichmäßig auf alle Protagonisten verteilt sind (Frederic: 9, Mabel: 9, Ruth: 4, General Stanley: 4) ${ }^{27}$ Betrachtet man sowohl die Häufung derartiger Liedformen als auch deren Inhalt (Verzicht auf tiefgründige Charakterisierung), dürften konsumorientierte Faktoren somit einen nicht unerheblichen Einfluss auf die Gestalt der Stücke gehabt haben. Die fassliche Gestalt und die lose Einbettung zwischen gesprochene Dialoge dürfte dabei mit Blick auf eine separate Veröffentlichung wohl kalkuliert gewesen sein, wie dies bereits von Komponisten des 18. Jahrhunderts beabsichtigt war. ${ }^{28}$ Die Frage der kompositorischen Realisisierung war aber auch eine Frage der Tradition. Obwohl Offenbach vor allem im Sinne seiner bisherigen Erfolge bei der Konzeption der Solonummern weiterhin auf die Form des Couplets vertraut, steht beispielsweise jenes des Falsacappa (Les Brigands I/4) entgegen den Versionen früherer Werke nicht mehr separiert, sondern ist hier nun in die Abfolge einer größeren Introduktionsnummer eingebunden. Offenbachs Interesse galt mehr und mehr komplexeren musikalischen Gebilden als der Komposition kurzer Lieder mit hübschen Refrains: "Je demande des situations à mettre en musique et non pas couplets sur couplets, comme dans TOTO ou LA DIVA. Le public se

still the music is generally good of its kind. We do not exactly see the connection with the tone and obvious idea of the story.", in: The Monthly Musical Record , 1. Mai 1880, S. 60

27 Als Solostücke wurden auch Couplets mit Chorbeteiligung gewertet, da dieser oft nur stützende Funktion besitzt. denen Anthologien, welche sich and musikalisch interessierte Laien wandten (z.B. "Vaudevilles et ariettes de l'Opéra Comique composé par $M{ }^{R}$ Corette", Paris o.J.) 
fatigue des petits refrains, et moi aussi." 29 Damit wird zugleich der Wunsch deutlich, eine dramatische Situation in der Musik weiterzuentwikkeln, sowie Musik- und Texteinheiten zu größeren szenischen Komplexen zusammenzuführen. Bei Gilbert und Sullivan hingegen herrscht noch die klassische Einlagenstruktur vor, mit regelmäßiger Abfolge gesprochenen und gesungenen Textes. Lediglich die Aktschlüsse gestalten beide im Sinne Offenbachs als vielgliedrige Szenenkomplexe. Die Spannweite des Liedbegriffs ist in beiden Fällen breit gefächert. So finden sich neben den Strophenliedern ebenso Arien, Buffolieder bis hin zur Ballade (The Pirates of Penzance II/28).

$$
-30-
$$

Ansatzweise ist der Versuch der Komponisten zu erkennen die musikalische Form dem sozialen Stand des vortragenden Akteurs anzupassen. So findet der Auftritt des Herzogs von Mantua in einem Duett mit Rondostruktur in einer kunstvolleren Form statt (I/5), während alle vorangegangenen Auftritte von Personen des Räubermilieus einfache Couplets waren. Ebenso darf Mabel ihre Qualitäten in einem Koloraturwalzer besingen, während Ruth nur ein einfaches Couplet zuerkannt wurde, wo zudem ein assistierender Chor fehlt.

$$
-31-
$$

Betrachtet man die Taktumfänge je Akt, so fällt auf, dass in beiden Werken jeweils der erste Akt den größten Anteil an Musik besitzt. Dies mag zum einen daran liegen, dass hier die für das szenische Verständnis wichtigen Auftrittsmusiken der einzelnen Handlungsträger lokalisiert sind (in beiden Werken je vier), welche in den folgenden Aufzügen entfallen. Zum anderen zeigt aber gerade das expandierte erste Aktfinale von Les Brigands, dass hier der Mittelpunkt des musikalischen Verlaufs, wenn nicht gar des gesamten Stückes zu suchen ist, denn der Choeur de Carabiniers (I/7b), die eigentliche Erfolgsnummer des Stückes, liefert das für die folgenden Finalensembles wesentliche motivische Grundmaterial, das sich am Schluss des Stückes als persiflierendes Zitat schließlich verselbständigt. Die Vermutung eines derart auf die Wirkung einer einzelnen Musiknummer abzielenden dramaturgischen Konzepts wird auch durch die Tatsache bestätigt, dass die Operette vor allem über diesen Chor rezipiert wurde. So findet sich ein ähnlich komponierter Chor auch in The Pirates of Penzance und stützt so zugleich die Vermutung, Sullivan habe die Partitur Offenbachs gekannt. Ebenso wie dort schreiten die völlig verängstigten, gleichwohl aber pflichtbewussten Bobbies in einem Marsch (II/17) zur Verhaf-

29 Brief Offenbachs an Ludovic Halévy vom August 1869, in Goninet, Lettres à Meilhac, S. 159. 
tung der Piraten an die Küste Cornwalls. Dieser ist ebenfalls mit Allegro marziale betitelt und nimmt zudem das Stiefelmotiv auf ("Un bruit de bottes"), indem die Polizisten um ihren Feinden Angst und sich selbst Mut $\mathrm{zu}$ machen, ihr Schuhwerk als Fanfaren benutzen ("Our heart is in the boots"), um gleichzeitig mit einem Schlachtruf ("Tarantara") ihrer zitternden Beine Herr zu werden.

$$
-32-
$$

Nahezu identisch ist die Häufigkeit im Auftreten bestimmter Ensembleformationen. Bei Offenbach sind es zwei Duette, ein Terzett und sechs Chornummern, in denen die Hauptfiguren in unterschiedlichster Besetzung den Chor bilden. Sullivans Werk besitzt die gleiche Anzahl an Duetten, drei Terzette und zwölf Chornummern. Eigenständig entwickelte Quartette und Quintette gibt es in beiden Werken nicht. Eine nennenswerte Divergenz ist die unterschiedliche Beteiligung des Chores. Während er in Les Brigands an 15 Musiknummern Anteil hat, tritt er in The Pirates of Penzance in elf Nummern in Erscheinung, was jedoch aufgrund der abweichenden Aktzahl nicht weiter von Bedeutung sein dürfte. Das häufige Auftreten der Chöre an sich steht im Zusammenhang mit der auf kompakte Gruppen ausgerichteten Personenkonstellation (Piraten, Polizisten, Hofstaat), welche sich naturgemäß mehrstimmig äußern. Generell kommt dem Chor in beiden Werken, über dekorative und kommentierende Funktionen hinaus, die Eigenschaft eines selbständigen Handlungsträgers zu. Beide Komponisten nutzen die Chöre zu komischen Effekten, einmal szenisch, um etwa durch numerische Expansion in Aktschlüssen mit Hilfe von Märschen und Massenbewegungen ein Durcheinander zu organisieren, oder artikulatorisch, indem durch einförmiges Auftreten (unisono) Einigkeit suggeriert wird. Daneben bestehen auch inhaltliche Parallelen, beispielsweise in Form des Alkoholgenusses. So rufen Gilberts Piraten zu Beginn im Trinkgelage ebenso fröhlich zum gemeinsamen Sherryumtrunk auf, wie die Mannen Falsacappas das Leeren der Branntweinfässer proklamieren. Unterschiedlich ist hierbei nur das Resultat. Während Meilhac und Halévy den Alkohol als inhaltlichen Movens benutzen, um ekstatische Zustände zu fördern, bleibt es bei Gilbert und Sullivan beim fröhlichen Zuprosten. Die Ensemblestücke, die furiosen Aktschlüsse zumal, erreichen in The Pirates of Penzance kaum den Grad an emotionaler Extrovertiertheit, mit denen Offenbach und seine Librettisten zeitweilig den gesuchten Rauschzustand in theatralische Form kleiden.

\section{$-33-$}

In der Wahl bestimmter Taktarten zeigen beide Komponisten eine eindeutige Präferenz von binären gegenüber ternären Metren. Dazu ist bei Offenbach eine Vorliebe für kleinmensurierte Takteinheiten festzustellen, da 
hier allein 18 Musiknummern im 2/4-Takt gesetzt sind. Vielleicht sollte auf diesem Weg die Charakterisierung der rohen und einfältigen Banditen geleistet werden. Hubert Stuppner dagegen bringt die Dominanz gerader Taktarten bei Offenbach mit einem 'Antriebsmedium der Beine' in Verbindung, in welchem eine "Spannungserzeugung im Zweitaktmechanismus von Laden und Entladen am augenscheinlichsten und primitivsten angelegt ist." ${ }^{30}$ Sullivan dagegen bevorzugt den 4/4-Takt (bzw. C), welcher in 15 Nummern gewählt wird. Kombinierte Taktarten und solche mit unsymmetrischer Zusammensetzung gerader und ungerader Einheiten erscheinen selten bis gar nicht. Dies dürfte unmittelbar damit in Zusammenhang stehen, dass die Stücke zumeist von singenden Schauspielern ausgeführt wurden und daher ein gewisses $\mathrm{Ma} \beta$ an Komplexität nicht überschritten werden durfte. Auch dem dilettierenden Liebhaber, welchem die Erfolgsnummern der Werke in separaten Anthologien angeboten wurden, sollte ein Nachmusizieren möglich sein. Eine bestimmte Tonartenarchitektonik ist nicht erkennbar. Die Spanne reicht bei Offenbach von E-Dur im Kreuztonartenbereich bis As-Dur im Bereich der B-Tonarten. Bei Sullivan sind darüber hinaus Abschnitte vorhanden, die jeweils ein Vorzeichen mehr haben. War schon die Abfolge der einzelnen Szenen merklich auf größtmögliche Abwechslung hin angelegt, gilt Entsprechendes anscheinend für den Bereich der Harmonik. Als bemerkenswertes Charakteristikum ist in The Pirates of Penzance lediglich die Häufung der mediantischen Verbindung einzelner Nummern zu erkennen (z.B. I/6 zu I/7; II/15 $\mathrm{zu}$ II/16). Ob die in Les Brigands zu beobachtende Aussparung von Tonarten mit mehr als vier Vorzeichen vorsätzlich im Sinne einer Popularisierung der Werke geschah, bleibt fraglich, da Offenbach in anderen Werken, die außerhalb dieser Betrachtung stehen, ebenfalls entlegenere Tonarten verwendet. Zudem ist davon auszugehen, dass einzelne Stücke, vor allem bei personeller Umbesetzung des Ensembles, den jeweiligen sängerischen Fähigkeiten angepasst und transponiert wurden. Somit bleibt als einzige verbindliche Gemeinsamkeit die Dominanz von Durtonarten. Abschnitte in Moll erscheinen nur akzidentiell (Les Brigands III/15a; The Pirates of Penzance I/2) und dienen ansonsten der kontrastierenden harmonischen Einfärbung innerhalb des tonalen Verlaufs einzelner Musiknummern. Die Vermutung, 'große' Taktarten könnten bei Offenbach höhergestellten Persönlichkeiten (spanischer Hofstaat) vorbehalten bleiben, bestätigt sich nicht, da beispielsweise auch die Gesänge der sozial niedriger einzustufen-

$30 \quad$ Hubert Stuppner: Technik und Ambivalenz musikalischer Lusterzeugung in Offenbachs Operetten, in: Jacques Offenbach (Musik-Konzepte 13), hg. v. Heinz-Klaus Metzger und Rainer Riehn, München 1980, S.54 
den Räuber in 6/8-Takten abgefasst sind (z.B. II/12). Deutlich wird bei Offenbach jedoch der Wille, über die Zuordnung bestimmter Tonarten zu einzelnen Personengruppen ein soziales Milieu zu kennzeichnen. So stehen etwa alle Auftrittslieder der Räuber im I. Akt in As-Dur (I/1b, I/2; I/4) und auch im II. Akt sind die - nun verkleideten - Banditen tonal eindeutig identifizierbar (II/11, II/12).

\section{Stichworte zur Orchesterbesetzung}

\section{Offenbach}

2 Flöten (2. auch Piccolo)

Oboe Oboe

2 Klarinetten

2 Fagotte

2 Hörner

2 cornet à pistons

Posaune

Pauke

Schlagzeug (gr.Tr, Trg, Bck)

Streicher

\section{Sullivan}

2 Flöten (2. auch Piccolo)

2 Klarinetten

Fagott

2 Hörner

2 Kornette

3 Posaunen

Pauke

Schlagzeug (gr. Tr, kl. Tr, Bck, Trg)

Streicher

$-34-$

Beide Komponisten vertrauen ihre Werke einem kleindimensionierten Theaterorchester an, mit doppelt besetzten Holz- und Blechbläsern sowie einer kleinstmöglichen Streichergruppe. Anders als Sullivan verwendet Offenbach jedoch die in französischen Oper- und Sinfonieorchestern des 19. Jahrhunderts (s. Berlioz) beliebten cornets à pistons mit einem helleren, durchdringenderen Klang. Die insgesamt sparsame Besetzung lässt einen durchsichtigen Orchesterklang erwarten, was sich beim Anhören der Stücke bestätigt und auch von Eduard Hanslick bei seinen Reisen so wahrgenommen wurde: "Das Orchester unterordnet sich bescheiden dem Gesang, ohne am rechten Ort auf lebhaftere Färbung und schärfere Charakteristik zu verzichten." 31

$$
-35-
$$

Die hierdurch angesprochenen klanglichen Effekte erreichten beide Komponisten vornehmlich durch ungewöhnliche Instrumentenkombinationen und figurierte Sonderparts, wenn etwa Holz- und Blechbläsergruppen ver-

31 Eduard Hanslick: Aus meinem Leben, Band 2, Berlin ${ }^{2} 1894$, S. 81. 
bunden wurden oder solistische Bläser zu einem ausgedünnten Streichersatz traten. Die geringe Größe des Orchesters erklärt sich wohl vor allem aus finanziellen Erwägungen. Beide Theaterunternehmungen standen unter enormen Konkurrenzdruck, so dass ein wirtschaftliches Überleben die Beschränkung der Personalkosten gebot. Zudem spielten aufführungspraktische Erfordernisse eine Rolle. Bedenkt man, dass es sich bei den Interpreten nicht um ausgebildete Opernsänger handelte, hätte ein allzu großer Orchesterapparat die wenig voluminösen Stimmen womöglich zugedeckt. Dem entspricht auch die in den Klavierauszügen häufig vermerkte Doppelung von Bass- oder Diskantlinien in der Begleitung, was eine Betonung hoher wie auch tiefer Frequenzen bewirken und die Tragfähigkeit des Gesanges unterstützen sollte. Dies wäre außerdem eine Reaktion auf die, Berichten zufolge, schlechten akustischen Verhältnisse in den Theatern selbst. ${ }^{32}$ Nicht zuletzt ermöglicht ein vom Umfang her kleines Orchester auch eine flexiblere Anpassung an das Bühnengeschehen, wenn etwa die Darsteller bestimmte spielerische Elemente hinzu improvisierten, oder es aufgrund der limitierten Gesangserfahrung einzelner Ensemblemitglieder zu Temposchwankungen kam.

\section{Zusammenfassung}

$-36-$

Obwohl vom Vorsatz her auf die Entwicklung eigenständiger Bühnenformen bedacht, verlassen beide Komponisten den von seriöser Theaterpraxis gesteckten Rahmen nicht. Beide Autorengespanne eint ein eklektizistischer Zugriff auf Elemente präexistenter Theatertraditionen. So zeigten die englischen Werke auf textlicher Ebene Einflüsse der Extravaganza, des Melodrams und der Burleske, während die Musik Traditionen von ballad opera und romantischer Oper fortschreibt und mit neuen Techniken Offenbachs mischt. Die französischen Libretti hingegen lassen Merkmale der Vaudeville-Komödien erkennen, während für Offenbachs Musik anscheinend der formale Bezug zur Opéra comique und die parodistische Hinwendung zur Grand Opéra wichtig war. In beiden Werken treten jedoch Musik, Sprache, Tanz und szenische Darstellung dergestalt in Verbindung, dass man die Werke insgesamt eindeutig dem musikalischen Vergnügungstheater zurechnen kann.

$$
-37-
$$

Die Analyse von Dramaturgie und Werkstruktur zeigte einerseits eine transparente Gesamtform im Kontext der Verbindung aus Dialog und Cou- 
plet, andererseits aber eine opernnahe Werkstruktur mit mehraktigem Aufbau, Bezügen zum Nummernprinzip und zeitweiliger Herausbildung umfangreicherer szenisch-musikalischer Komplexe. Die Verbindung dieser selbstständigen musikalischen Einheiten lösen beide Komponisten jedoch unterschiedlich. Während Sullivan auf das Rezitativ zurückgreift und somit das bewährte Schema einer geschlossenen Opernnummer fortführt, bedient sich Offenbach des Melodrams, welches in Frankreich weitaus populärer war als in England und in der Mitte des 19. Jahrhunderts in der Opéra comique äußerst beliebt war.

\section{$-38-$}

Das Auftreten bestimmter Gesangsmodelle ließ eine Differenzierung in solche solistischer oder gemeinschaftlicher Form erkennen. Dabei dienten die solistischen Formen der Exponierung von Handlungsträgern und der Retardierung des Handlungsverlaufs, während die Ensembleformationen zu einem Großteil szenische Aktion vermittelten und die periodisch wiederkehrenden Konfliktsituationen musikalisch umrahmten. Deutlich wird bei Offenbach jedoch der Wille, diese Konfliktsituationen im Gegensatz zu Sullivan besonders in den Aktschlüssen auf eine ekstatische Massenszene hin zuzuspitzen, wobei die intendierte Übertragung eines motorischen Bewegungsimpulses auf das Publikum sehr deutlich wird. Eine besondere Aufwertung erfuhr bei beiden Komponisten der Chor, der gegenüber seiner Verwendung in theatralischen Kleinstformaten wie Posse mit Gesang, Burleske oder Liederspiel zu einem selbstständigen Handlungsträger avancierte. Ihm oblag es nicht nur zu Beginn der einzelnen Aufzüge, die atmosphärische Grundstimmung zu setzen, sondern er trat darüber hinaus als eigenständiger Identitätsträger auf, verstärkte Emotionen, antizipierte oder wiederholte Refrains und wurde in den Auftrittsliedern gar zum Dialogpartner.

$$
-39-
$$

Die Texte beider Autoren zeichnen sich durch eine besondere literarische Qualität aus, wobei die Dialoge geistreich, satirisch und mit Wortspielen gespickt sind und bei stets graziler Ironie für die entsprechende Situation meist das treffende Wort finden. Vor dem Hintergrund, eine nationale komische Oper zu etablieren, verzichtet Gilbert jedoch zum Zwecke der Abgrenzung auf jegliche Frivolitäten und Zweideutigkeiten. Hierin kommt einerseits eine unterschiedliche Sozialbindung beider Künstler zum Ausdruck (libertinäres Frankreich - puritanisches England), andererseits wird damit einem unterschiedlichen Unterhaltungsbedürfnis des Publikums in Paris gegenüber dem in London entsprochen, wo Wortwitz ('wit'Phänomen), Nonsense-Logik und schwarzer Humor bis heute als typische Konstanten britischen Amusements gelten. 
Auch in der Musik werden diese divergierenden musikästhetischen Prämissen deutlich. Die Analyse des Verhältnisses von Wort und Musik zeigte, dass Sullvan letzterer insgesamt mehr Raum gibt und somit seine Werke offensichtlich eher als komische Opern denn musikalisierte Schauspiele verstanden wissen wollte. Differenzen werden auch bei der Darstellung musikalischer Komik deutlich. So enthält sich Sullivan jeglichen musikalischen Klamauks und verfährt in einer Art und Weise, die sich im Vergleich zu Offenbachs Neugier und Experimentierfreude weitaus gediegener ausnimmt. Letzterer wusste offenbar um die Rezeptionsmechanismen am Pariser Boulevard und suchte im verfeinerten Zugriff auf alle zur Verfügung stehenden Mittel das Bedürfnis nach Neuem mit kompositorischen Extravaganzen zu befriedigen. 